\title{
$2 \times 2$ MEMS FIBER OPTIC SWITCHES WITH SILICON SUB-MOUNT FOR LOW-COST PACKAGING
}

\author{
Shi-Sheng Lee, Long-Sun Huang*, Chang-Jin "C.J" Kim* and Ming C. Wu \\ Electrical Engineering Department, UCLA \\ 63-128, Engineering IV Building, Los Angeles, California 9()(095-1594 \\ *Mechanical and Aerospace Engineering Department
}

\begin{abstract}
We report on a novel $2 \times 2$ MEMS fiber optic switch. The switch is implemented by ganging up multiple vertical torsion mirror devices that are fabricated using silicon surface micromachining process. Bulk-micromachined silicon submount has been developed for the switch. The submount is designed to accommodate the surface-micromachined vertical torsion mirrors (mirror chip). micro lenses and optical fibers. This new hybridpackaging method is used to minimize active optical alignment between the mirror chip and optical fibers. thus reduce the packaging cost of the switch. Sub-millisecond switching times have been achieved. With this hybrid-packaging scheme, the size. weight. and potentially the cost of current fiber optic switches can be dramatically reduced.
\end{abstract}

\section{INTRODUC'TION}

There has been a significant growth of optical fiber networks due to the need of fast, broadband local area networks. The rapid growth of fiber-based local area networks has created a large demand for low-cost fiber optic switches. Fiber optic switches are used to reconfigure the network and/or increase its reliability. For example. the FDDI (Fiber Data Distribution Interface) fiber optic ring network employs optional $2 \times 2$ fiber optic switches. called optical bypass switches. to bypass the failed nodes thus preserve the network reliability.

There has been a growing interest in applying MEMS (Micro-Electro-Mechanical System) technology to reduce the size. weight. and cost of the opto-mechanical switches [1-3]. Micromachined torsion mirrors are very attractive for implementing optical switches because of therr fast switching time and proven reliability [4]. However. conventional torsion mirrors with in-plane torsion beams present a great challenge for packaging since the optical beams are scanned in the out-of-plane direction. Recently. we have reported a novel vertical torsion mirror switching device [5] whose torsion beam is perpendicular to the substrate. Such vertical torsion mirror is particularly suitable for implementing fiber optic switches since the mirror remains perpendicular to the substrate at all scanning angles.

In this paper. we report a novel $2 \times 2$ MEMS fiber optic switch that consists of four vertical torsion mirror devices. In addition. a new hybrid packaging scheme has been developed using bulk-micromachined silicon submount for low cost manufacturing of the switches. The torsion mirror has a pull-in voltage of $83.5 \mathrm{~V}$. Sub-millisecond switching times have been achieved. The detail design and fabrication of the switch will be presented in the following sections.

\section{DESIGN AND FABRICATION}

Scanning electron micrograph (SEM) of the switch is shown in rig. 1. The switch consists of a mirror chip and a silicon submount. The mirror chip comprising four surfacemicromachined vertical torsion miror devices is passively integrated with the silicon submount. The principle of switch uperation is illustrated in Fig. 2. The four vertical torsion mirror devices are arranged such that in the REFI.ECTION mode, the input beams are reflected by two 45 -degree vertical torsion mirrors and coupled into the output fibers located on the same side of the chip. In the TRANSMISSKON mode, the vertical torsion mirrors are rotated out of the optical paths, and the input beams are coupled into the opposite output fibers.

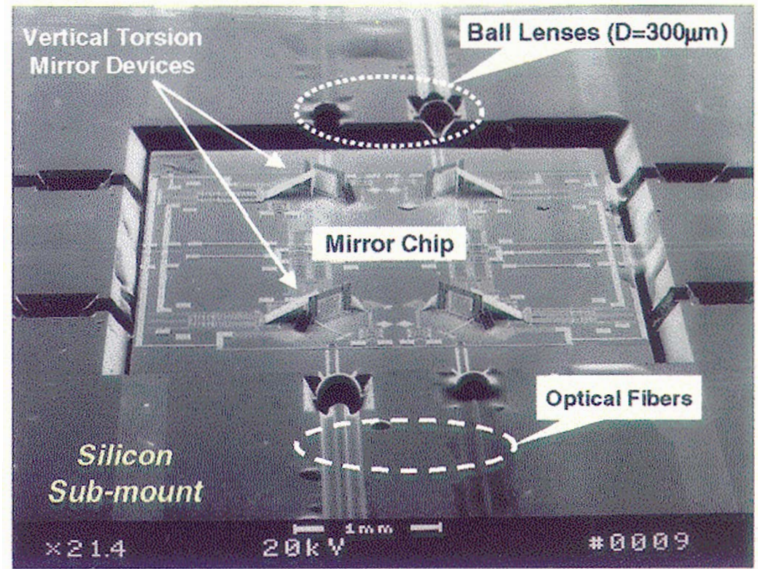

Figure 1. SEM of the $2 \times 2$ MEMS fiber optic switch.

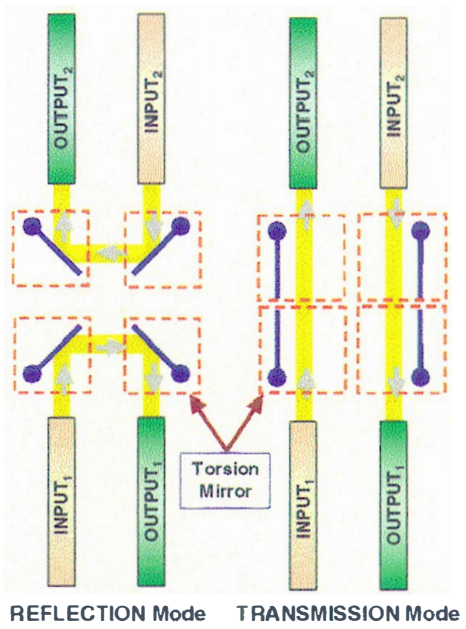

Figure 2. Schematic: illustrations of the principle of operation.

The basic microstructures of the mirror chip is fabricated by the three-layer polysilicon surface-micromachining process at MEMS Technology Application Center at North Carolina (MCNC) under the Multi-User MEMS Processes (MUMPs) service. Additional in-house processes have been done in order to improve 
both mechanical and optical characteristics of the switch. The submount is fully designed and fabricated at UCL.A.

\section{Mirror Chip}

The mirror chip is made of four vertical torsion minror switches. A torsion mirror device consists of a vertical torsion mirror and a vertical back electrode plate. The vertical back electrode is employed to electrostatically bias the mirror plate. Buth the vertical torsion mirror and the back electrode are realized by the micro-hinge technology $|6|$. The angle between the vertical torsion mirror and the back electrode is designed to be $45^{\circ}$. Figure 3 shows SEM of a torsion mirror device. The back electrode plate is integrated with a scratch drive actuator (SDA) [7] array for selfassembly purpose. This self-assembly approach could dramatically reduce total assembly time of the switch. It is particularly important when multiple vertical torsion devices are used to implement more sophisticated functions. In principle. the vertical torsion mirror can also be self-assembled.

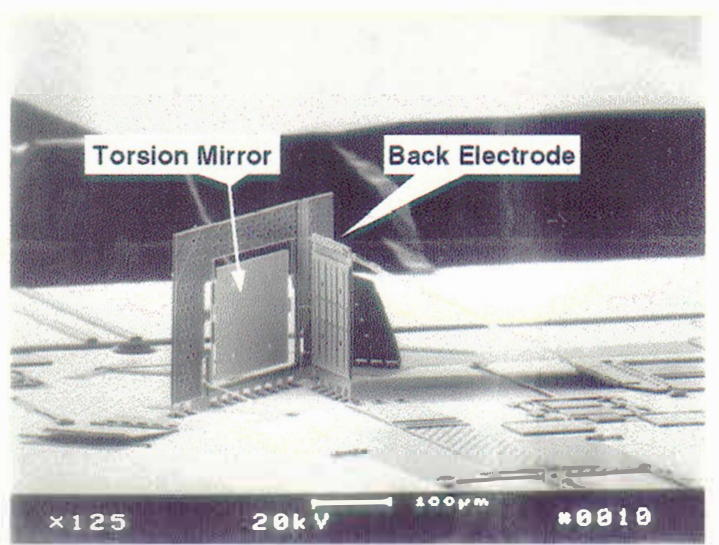

Figure 3. SEM of the torsion mirror device.

Vertical torsion mirror consists of a torsion mirror and a vertical-supporting frame. SEM of the vertical torsion mirror is shown in Fig. 4. The mirror is connected to a fixed vertical frame through a torsion beam. A $6 \mu \mathrm{m}$-wide and $2 \mu \mathrm{m}$-thick polysilicon stopper beam is integrated with the supporting frame to prevent overshoot of the mirror plate when it is restored by the torsion beam. The minor plate is $200 \mu \mathrm{m}$-wide, $160 \mu \mathrm{m}$-tall and $1.5 \mu \mathrm{m}$ thick. The torsion beam is $2.0 \mu \mathrm{m}$-wide, $78 \mu \mathrm{m}$ long and $0.8 \mu \mathrm{m}$ thick. The surface of the mincor is coated with $0.1 \mu \mathrm{m}$-thick gold layer to improve the optical reflectivity. The micro-hinge allows

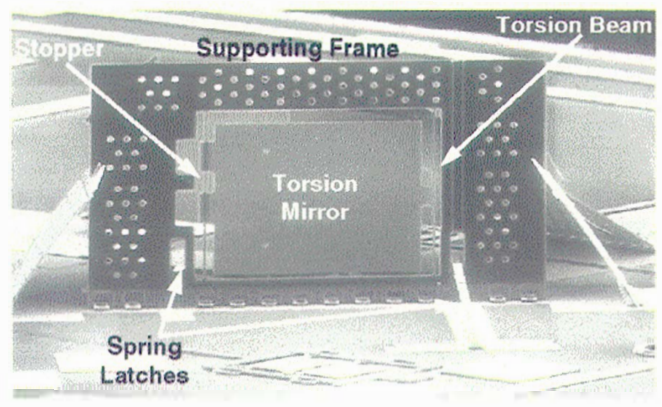

Figure 4. SEM of the vertical Iorsion mirror:

the supporting frame to rotate out of the $\mathrm{Si}$ substrate and stand perpendicular to the substrate. The frame is fixed by two side support plates and a pair of spring latches. The two spring latches are anchored to the opposite sides of the frame to cancel out possible excess free movement of the plate and to minimize the separation between the assembled mirror and the back electrode. The side support plates minimize the motion of the frame during the switch operation. Many large etching holes are used on the frame to reduce the electrostatic interaction between back electrode and the frame.

Vertical back electrode is made of three polysilicon plates (a back electrode plate, a support plate. and an actuator plate for selfassembly) that are jointed together by micro-hinges as shown in Fig. 5. When the electrode is assembled by the SDA. it forms a triangular shape from the side view. This configuration strengthens the stability of the back electrode during the switch operation. The total displacement of SDA anray is designed such that the back electrode will be perpendicular to the wafer when SDA array is fully actuated. To prevent direct contact with the mirror during switch operation. a micro-stopper has been employed.

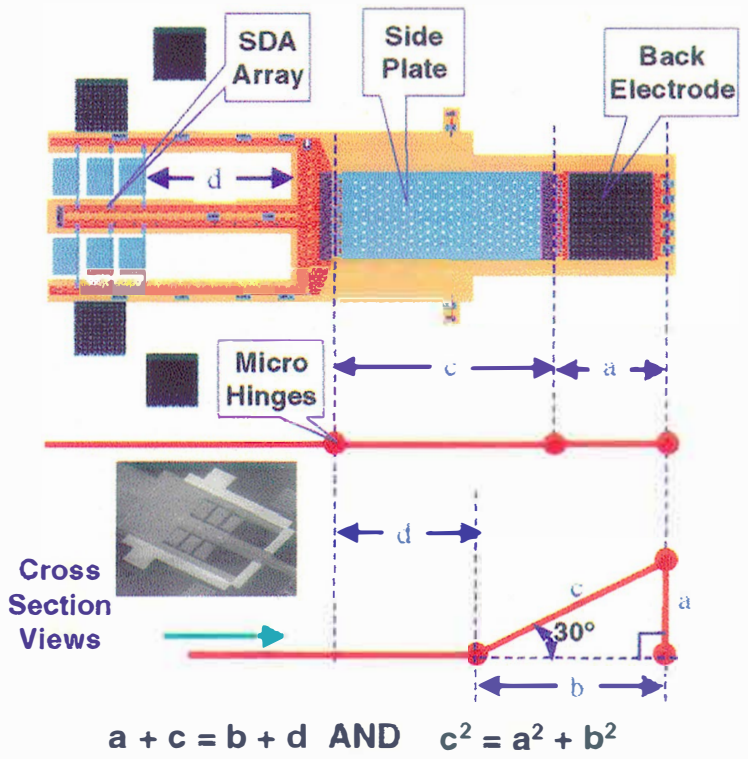

Figure 5. Schematic drawing of the back electrode.

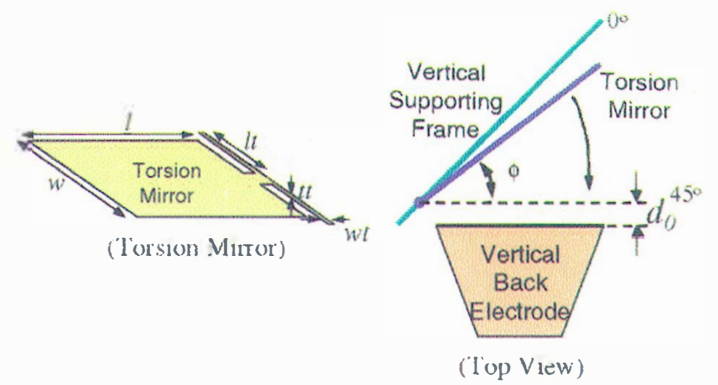

Figure 6. Geornetrical relations between the mirror and back electrode.

The switch is designed to have a switching voltage of less than $100 \mathrm{~V}$. Figure 6 shows the geometrical relation between the mirror and the back electrode. The electrostatic torque (Te) exerted by the applied voltage and the restoring torque (Tr) of the torsion beam can be expressed as functions of the rotation angle $\phi$ ( $\phi$ can vary from ()$^{\circ}$ to $45^{\circ}$ ) [1]. where $G$ is the shear modulus of polysilicon. 


$$
\begin{aligned}
& \mathrm{Tr}=\frac{2}{3} \cdot \frac{\left(\dot{s} \mathrm{w} t \cdot \mathrm{tt}^{3} \cdot 0\right.}{\mathrm{tt}} \cdot\left(1-\frac{192}{\pi^{5}} \cdot \frac{\mathrm{t}}{w \cdot t} \cdot \tanh \left(\frac{\pi \cdot w \mathrm{t}}{2 \cdot \mathrm{tl}}\right)\right)-(1) \\
& \mathrm{Te}=\frac{\varepsilon \cdot \mathrm{w} \cdot V^{2}}{2} \cdot \int_{0}^{1} \frac{x}{\left[\left(\frac{\pi}{4}-0\right) \cdot x+\mathrm{do}\right]^{2}} \mathrm{dx}-(2)=
\end{aligned}
$$

Since the restoring torque is roughly proportional to the third power of the torsion beam thickness. reducing the thickness is the most effective way to reduce the operating voltage without increasing the size of the switch. Here. the thickness of the torsion beam is reduced from $1.5 \mu \mathrm{m}$ to $0.6 \mu \mathrm{m}$ by reactive ion etching (RIE) with $\mathrm{SF}_{6}$ gas. which yields a pull-in voltage of $83.5 \mathrm{~V}$. No change of the mechanical strength of the torsion mirror plate is observed after etching.

\section{Silicon Submount}

The packaging scheme of the switch using silicon submount is to achieve a pick-and-drop type of hybrid packaging. Figure 7 shows the cross-section view of the package - a free-space micro mirror chip placed in the silicon submount. The submount consists of two bulk-micromachined silicon substrates bonded together.

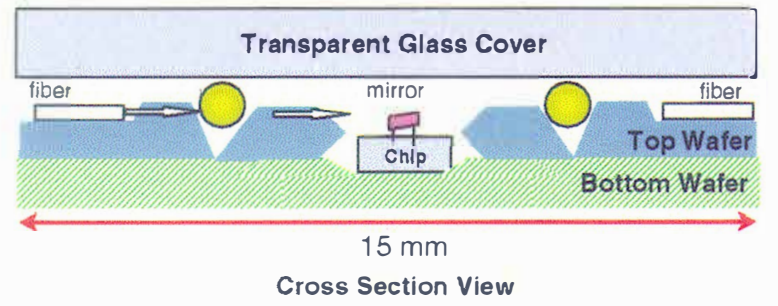

Figure 7. Cross-section view of the package. a mirror chip placed on Si submount and covered.

The top wafer of the Si submount has a central opening for the mirror chip. V-grooves for optical fibers, and micropits for micro ball lenses. The bottom substrate has a central recess area and is bonded to the top substrate. With two-substrate approach. free-space M()EMS (Micro-(.)ptical-Electro-Mechanical Systems) devices of different designs can be accommodated with only minor modification. With this hybrid packaging scheme. the mirror chip and the silicon submount can be fabricated and optimized separately. thus avoiding the complexity associated with fabricating all the features on a single chip. The packaged mirror chip can then be easily packaged into a final product.

For the switch. light emitted from the input fiber is transmitted to the opposite fiber or redirected by the mirror to the fiber on the same side. Micro ball lenses are used to enhance optical coupling efficiency and increase working distance. In order to assemble optical elements. the silicon submount is required to have proper mechanical structures to hold the optical components. The silicon structures required for optical elements on the submount are $\mathrm{V}$-grooves for fibers. micropits for ball lenses, and a central recess for a miror chip. The V-grooves, micropits and central recess can be realized by anisotropic wet etching of $(10)(0)$ silicon wafer. However, it is known that convex corner on a $(10))$ silicon wafer is attacked during an anisotropic wet etch process making formation of convex corners difficut. Therefore. carefully designed corner compensation structures [8] are used to preserve convex corners during the wet etching process
Figure 8 shows the fabrication procedures. Two (100) silicon wafers are used for the top (double polished) and the bottom substrates. First. a 100)A-thick silicon dioxide is thermally grown, followed by a 150)A-thick I.PCVD (low-pressure chemical-vapordeposition) silicon nitride layer as a silicon-etching mask during the anisotropic wet etching. To minimize the effect of silicon crystal lattice-to-mask misalignment during the etching. determination of the true crystal direction within (). $1^{\bullet}$ accruacy [9] is desirable for the top wafer. Reference marks are etched to reveal the true crystal direction at the beginning of the process. After using reactive ion etching ( $\mathrm{RIE})\left(\mathrm{CF}_{4} / \mathrm{O}_{2}\right)$ to open up all etch windows on the both sides of the top wafer. the top wafer is etched in $\mathrm{K}() \mathrm{H}$. (Once the central hole is opened. the wafer is taken out of the etching solution. All the features are fabricated in a single anisotropic wet etching step. Then a $500(0) \AA$-thick aluminum pad is deposited for wire bonding by the lift-off process. The bottom silicon substrate is prepared by a simpler process to create the recess for the switch chip.

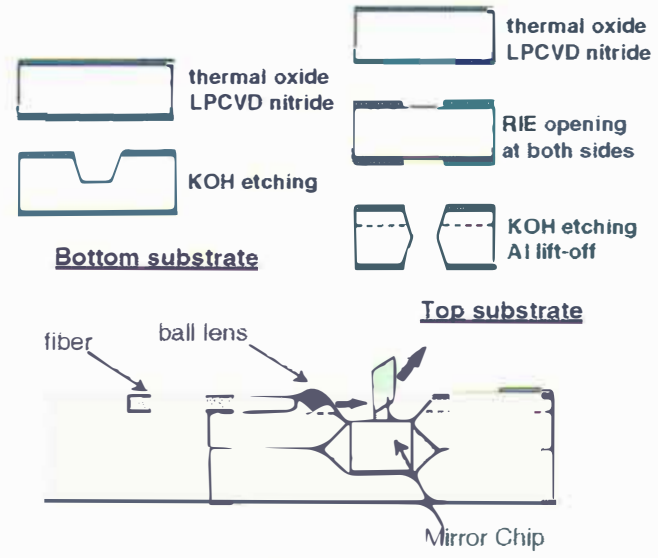

Figure 8. Process flow of Si submount.

\section{Assembly}

Figure 9 shows the schematic drawing of the silicon submount with optical fibers and micro ball lenses in place. First. the top and bottom wafers are diced to the size of $15 \mathrm{~mm} \times 15 \mathrm{~mm}$. In order to align top and bottom substrates. dummy optical fibers with diameter of $125 \mu \mathrm{m}$ are used. These optical fibers are placed in the channels that are formed by two V-grooves in the interface of top and bottom substrates. Thermally cured adhesive applied along the perimeter of the bottom surface bonds the two substrates together. (Orthogonally arranged dummy fibers lock the two substrates against translational and rolational sliding during the curing step when pressure is applied to the two mating substrates. Then. the mirror chip is dropped in the center opening and 30) $\mu \mathrm{m}$-diameter ball lenses are picked and dropped into the micropits. Finally, four optical fibers are placed in V-grooves and glued using UV epoxy. Figure 10 shows the assembly of the fiber and ball lens on the V-groove and micropit.

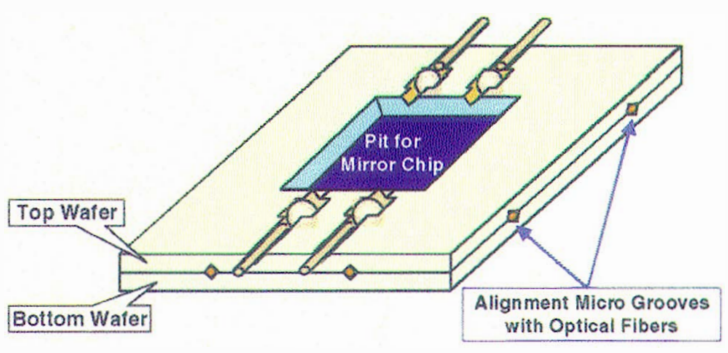

Figure 9. Schematic illustration of the silicon submount. 


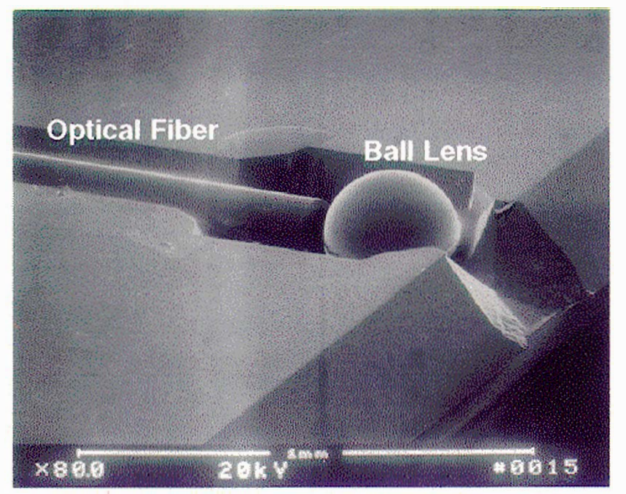

Figure 10. SEM of the fiber and ball lens assembly.

\section{EXPERIMENTAI, RESUITS}

The do switching characteristics is plotted in Fig. 11. which shows the measured angle of the mirror versus the applied $D C$ voltage. A pull-in voltage of $83.5 \mathrm{~V}$ and a releasing voltage of 53 $V$ have been achieved. The plot clearly shows the hysteresis that is characteristic of an electrostatic gap-closing actuator. The pull-in voltage and releasing voltage can be optimized further by adjusting the thickness of the torsion beam and the area of the back electrode. Electrical insulation between the mirror and the back electrode after pull-in is achieved by a mechanical stopper.

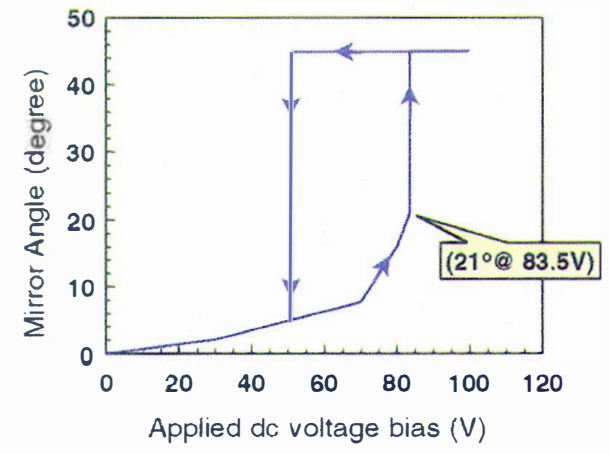

Figure 11. DC switching characteristic:

The dynamic response of the switch is plotted in Fig. 12 TRACE 1 corresponds to the ()$^{\circ}-10-45^{\circ}$ switching by the electrostatic torque. and TRACE 2 corresponds to the $45^{\circ}-10-()^{\circ}$ switching by the restoring torque of torsion beam. Switching time (including the settling time) of less than $1 \mathrm{~ms}$ has been achieved. We have observed some oscillations after the initial switching. We believe the uscillations are due to bending of the torsion beam and overshoot during impact with the micro-stopper. This effect can be minimized by modifying mirror structure or using a programmed voltage to slow down the impact speed of the mirror during the switching thus reduce the mechanical oscillation. Eliminating the oscillations can further reduce the switching time.

Before mounting the switch on the silicon submount, the optical insertion loss is measured to be $1 \mathrm{~dB}$ with active optical alignment and a working distance of $6(0) \mu \mathrm{m}$. With passive optical alignment on the silicon submount. due to a relatively large mirror chip size. optical coupling loss is on the order of $10 \mathrm{~dB}$ (includes Fresnel loss and spherical aberration loss). It is possible to achieve an optical coupling loss of less than $2 \mathrm{~dB}$ (includes fresnel loss and spherical abenation loss) by reducing the mirror chip to less than $1 \mathrm{~mm}$ in size. The crosstalk of the switch is less than -6() $\mathrm{dB}$ (measurement limit).

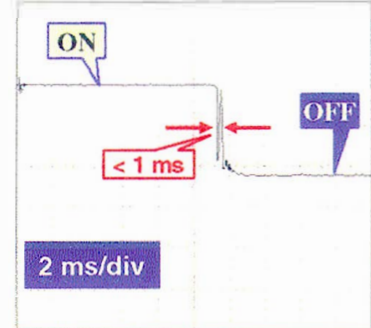

TRACE 1

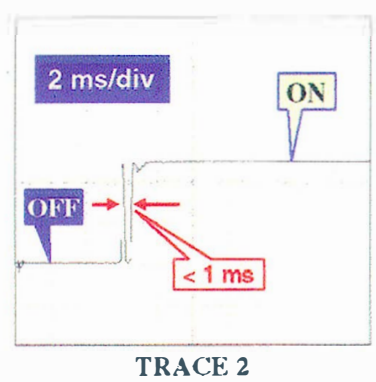

TRACE 2
Figure 12. Dynamic response of the switch.

\section{CONCI.USION}

We have demonstrated a 2x2 MEMS fiber optic switch based on vertical torsion mirrors fabricated by the surface micromachining process. A new bulk micromachined silicon submount has been developed for optical packaging of the switch. The submount is used to minimize active optical alignment between the mirror chip and optical fibers. thus reduce the packaging cost of the switch. Optical coupling loss is relatively high for the current device, however, the coupling loss can be further reduced by reducing the size of the mirror chips. Submillisecond switching times have been achieved. With this hybridpackaging scheme, the size. weight. and potentially the cost of current fiber optic switches can be dramatically reduced.

\section{REFERENCE}

1. H. Toshiyoshi and H. Fujita, "Optical crossconnection by silicon micromachined torsion mirrors", Digest of IEEE/L.EOS 1996 Summer Topical Meetings, pp. 63-64, Keystone, CO. August, 5-9, 1996.

2. C. Marser, M.-A. Gretillat, N. F. de Rooji. et al, "Vertical mirrors fabricated by reactive ion etching for fiber optical switching applications". Proc. 10th IEEE International MEMS Workshop. pp. 349-54, Naguya. Japan, January 26-30. 1997.

3. S. S. Lee and M. C. Wu, "Surface-micromachined free-space fiber optic switches with integrated microactuators for optical fiber communication". International Solid-State Sensors and Actuators Conference - TRANSDUCERS '97, pp. 85-88. Chicago. II. June 16-19. 1997.

4. L. J. Hornbeck. "Digital light processing and MEMS: an overvicw", Digest. IEEE/LEOS 1996 Summer Topical Meetings, pp. 7 . 8. Keystone, (O), August 5-9. 1996.

5. S. S. I.ee and M. C. Wu, "Surtace-micromachined vertical torsion mirror switches". Lnternational Conference on Optical MEMS and Their Applications-MOEMS 97. pp. 115-118. Nara, Japan. Nov. 1821, 1997

6. K. S. J. Pister, M. W. Judy. S. R. Burgett, and R. S. Fearing, "Microfabricated hinges", Sensors and Actuators A-Physical, Vol. 33, no. 3, pp. 249-256. 1992.

7. T. Akiyama and $\mathrm{H}$. Fujita, "A quantitative analysis of scratch drive actuator using buckling motion", Proc. $8^{\text {th }}$ IEEE International MEMS Workshop. pp. 310-315, Amsterdam, Netherlands, January 29February 2, 1995.

8. 1..-S. Huang, S. S. Lee, E. Motamedi. M. C. Wu and C.-J. Kim. "MEMS packaging for micro mirror switch", 48 Components \& Technology Conference. Seattle, WA, May 25-28. 1998 (to be appeared).

9. G. Ensell, "Alignment of mask patterns to crystal orientation". Proc: gh $^{\text {th }}$ International Conference on Solid-State Sensors and Actuators - TRANSDUCERS'95, Eurosensors IX. pp. 186-189.vol. 1. Stockholm. Sweden. June 25-29.1995. 